\title{
A Synchrotron Radiation Photoelectron Spectroscopic Study on the Oxidation of Si in Diamond-like Carbon Film by Hyperthermal O-atom Beam
}

\author{
Kumiko Yokota, ${ }^{1} *$ Masahito Tagawa, ${ }^{1}$ Akitaka Yoshigoe $^{2}$ and Yuden Teraoka ${ }^{2}$ \\ ${ }^{1}$ Kobe University, Rokko-dai 1-1, Nada, Kobe 657-8501, Japan \\ ${ }^{2}$ Japan Atomic Energy Agency, Kouto1-1-1, Sayo, Hyogo, 679-5148, Japan \\ *yokota@mech.kobe-u.ac.jp
}

(Received : November 11, 2013; Accepted : January 9, 2014)

\begin{abstract}
Oxidation reaction efficiency of the embedded Si atoms in the diamond-like carbon (DLC) film was studied with a combination of the broad O-atom beam, high-speed chopper wheel and synchrotron radiation photoelectron spectroscopy (SR-PES). The high-speed chopper wheel converted the translational energy distribution of O-atom into spatial distribution on the DLC surface. High spatial and energy resolutions of SR-PES allow studying the difference in oxidation states of $\mathrm{Si}$ atoms in different translational energies. It was confirmed that the $\mathrm{SiO}_{2}$ was formed by the high-energy collision conditions of O-atoms, whereas sub-oxides are formed with the low-energy collisions. The efficiency of $\mathrm{SiO}_{2}$ formation at $9 \mathrm{eV}$-collision is evaluated to be 4 times greater than that at $2 \mathrm{eV}$-collision.
\end{abstract}

\section{Introduction}

There exist many environmental factors in low Earth orbit (LEO) such as micro-gravity, thermal cycling, plasma, ultraviolet, radiation, neutral gas and space debris. In particular, one of the most important factors that gave serious damage to the many polymeric materials is $\mathrm{O}$-atom which is a dominant neutral species in LEO. Due to its high chemical reactivity and high impact energy, O-atom in LEO erodes many materials used in the exterior surface of spacecraft especially carbon-based materials such as polymers and diamond-like carbons (DLCs) [1-3].

Embedded $\mathrm{Si}$ atoms in the main chain of polymer is a promising technology to protect polymeric materials from a harsh space environment in LEO. When Si atoms in the main chain of polymers are oxidized by O-atom bombardment in LEO with collision velocity of $8 \mathrm{~km} / \mathrm{s}$ (collision energy of $5 \mathrm{eV}$ ), it is expected that the non-volatile $\mathrm{SiO}_{2}$ layer was formed at the surface. The formation of $\mathrm{SiO}_{2}$ layer at the polymer surface in LEO provides the capability of self-healing protective materials against O-atom attack in LEO [4]. Therefore, understanding of the oxidation reaction of O-atom with $\mathrm{Si}$ with collision energies in a few electron volts is important for space engineering.

On the other hand, the oxidation of $\mathrm{Si}$ at low temperatures is also crucial as a semiconductor fabrication technology. Many of such low temperature processes use oxygen plasma which includes O-atoms as an oxidant. However, the oxidation reaction of $\mathrm{Si}$ by O-atom has not been fully understood through experiments. One of the reasons is the difficulty of creating a high-density O-atom environment without any byproduct. Namely, oxygen plasma usually involves not only O-atoms, but also excited $\mathrm{O}_{2}$ molecules and ions. The presence of such chemically reactive byproducts makes the analysis of the reactivity of O-atoms difficult. Therefore, a high-purity $\mathrm{O}$-atom environment is necessary to study the oxidation reaction of $\mathrm{O}$-atoms with $\mathrm{Si}$. 


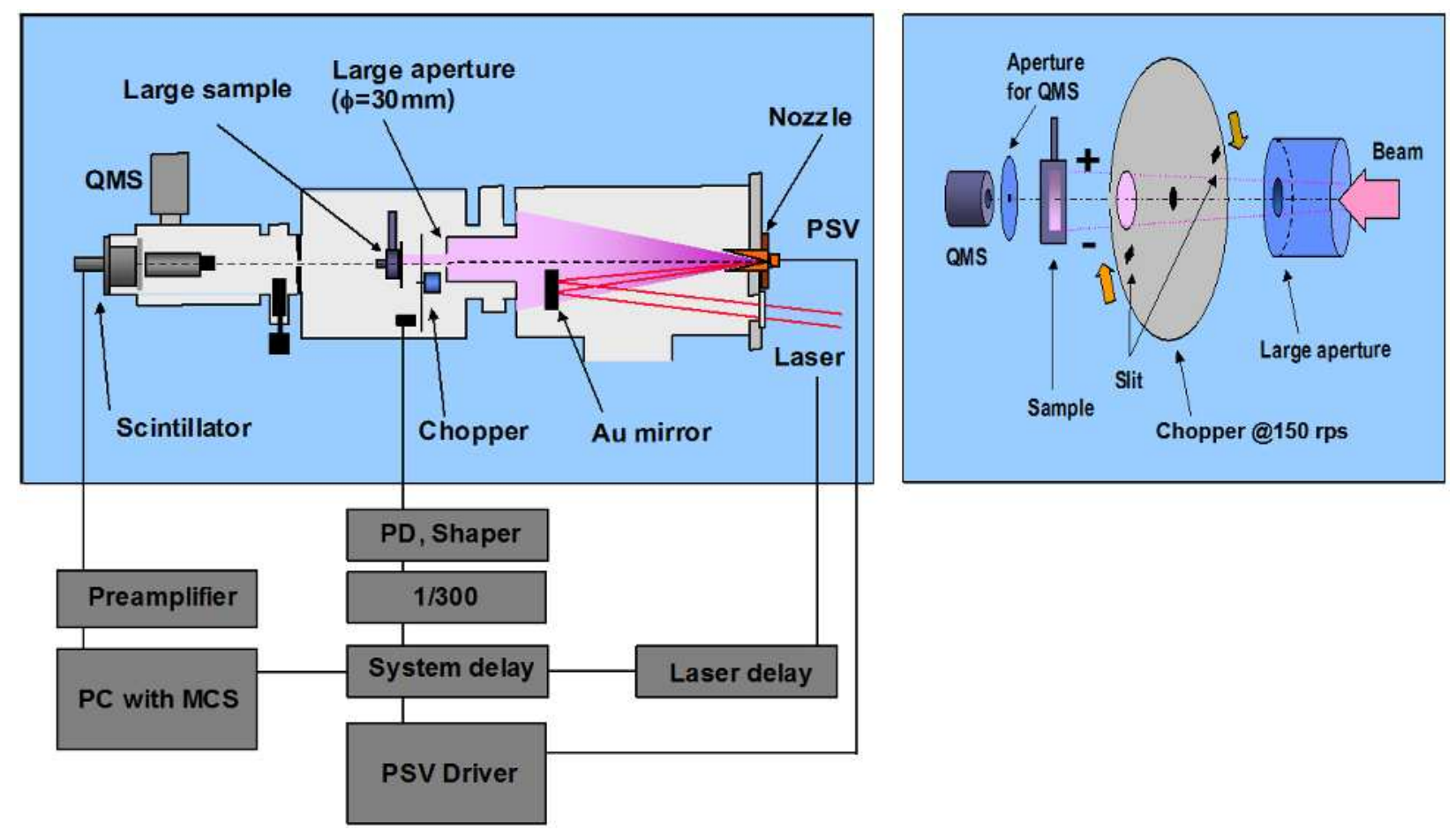

Fig. 1 The system diagram and detail of the velocity- position conversion by the chopper wheel. The O-atom beam with diameter of $30 \mathrm{~mm}$ was sliced by the chopper wheel rotating $150 \mathrm{rps}$ (two slits on the disc). A photodiode signal (300 $\mathrm{Hz})$ was reduced to $1 \mathrm{~Hz}$ and used as a system clock. Because of the $\mathrm{CW}$ rotation of the wheel, the negative positions on the surface are high-energy exposure positions.

In this study, energy dependence in oxidation reaction of $\mathrm{Si}$ atoms in DLC films was studied by the hyperthermal O-atom beam condition which simulates space environment in LEO. It has been known that the O-atom beam produced in this method contains small amount of undecomposed $\mathrm{O}_{2}$ and almost no ion $[5,6]$. It is much more pure O-atom environment than in $\mathrm{O}_{2}$ plasma. Thus, the data obtained in this study would be useful for understanding of the oxidation reactions in O-atom and Si system.

\section{Experimental Details}

The hyperthermal O-atom beam was produced by the laser-detonation facility which has been used for simulating high-energy $(5 \mathrm{eV})$ collision phenomena of O-atom with materials in LEO (Fig. 1). The detail of the facility was reported elsewhere [6]. A combination of the broad O-atom beam generated by the laser-detonation method and the high-speed mechanical chopper wheel (150 rps) was applied in this study. This combination could convert a velocity distribution of the O-atom beam into a spatial distribution on the specimen surface. The time-of-flight (TOF) distributions at the sample position from -6 to $+12 \mathrm{~mm}$ from the beam axis are shown in Fig. 2. Position “ \pm 0 ” represents the center position of the sample (on the beam axis). The TOF distributions at the out-of-axis positions were measured by the on-axis QMS at the same slicing timing. Difference in spatial distribution on the TOF distributions was ignored since the $12 \mathrm{~mm}$ on the sample surface corresponds to less than $1^{\circ}$ in the viewing angle from the nozzle throat. It was analyzed from these TOF spectra that the collision energy of $\mathrm{O}$-atom distributed from 2 to $9 \mathrm{eV}$ depending on the location of the specimen. The flux variation with energy in the sliced beam was compensated by the intensity of TOF distribution of the sliced beam. Figure 3 shows the energy and intensity distributions over the sample surface. It was confirmed that the maximum flux was obtained at the location of +4 $\mathrm{mm}$ position, where the average $\mathrm{O}$-atom energy is $2.5 \mathrm{eV}$. The negative positions are exposed to the O-atom beam with low-flux but high-energy. The Si-DLC sample (15 $\mathrm{mm} \times 30 \mathrm{~mm}$ ) was cleaved from a DLC-coated Si wafer which was prepared by CVD method. The DLC layer 
K. Yokota et al. A Synchrotron Radiation Photoelectron Spectroscopic Study on the Oxidation of Si in Diamond-like Carbon Film by Hyperthermal O-atom Beam
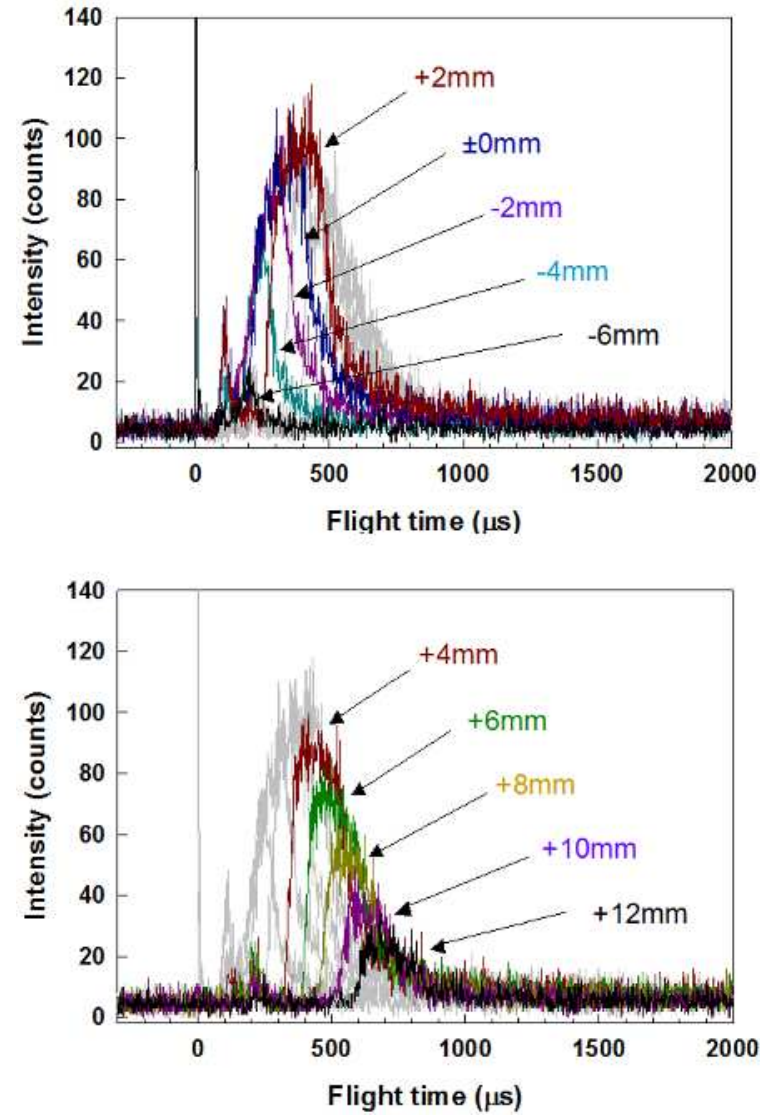

Fig. 2 Sliced TOF distributions of O-atom at the sample positions relative to the center axis of the apparatus. The TOF spectra of the O-atom at high-energy positions on the sample are shown in the top panel. Those at low-energy positions are shown in the bottom panel. The time zero in the abscissa represents the moment of laser fires.

was $1 \mu \mathrm{m}$ in thickness and $\mathrm{Si}$ content was approximately 10 at\%. The hydrogen content of the sample estimated by the elastic recoil detection analysis (ERDA) is roughly 30 at $\%$, respectively.

The synchrotron radiation photoelectron spectroscopy (SR-PES) measurements were performed by the SUREAC2000 end-station at the BL23SU in the SPring-8 facility. The SR-PES measurements were carried out with X-ray energy of $849.7 \mathrm{eV}$. All SR-PES spectra were taken with the take-off angle of $90^{\circ}$ (surface normal).

\section{Results and Discussion}

Figure 4 shows the SR-PES O1s and Si2p intensity distributions over the sample surface. O1s intensity shows the summit between -6 and $+2 \mathrm{~mm}$ where relatively low-flux and high-energy positions. This

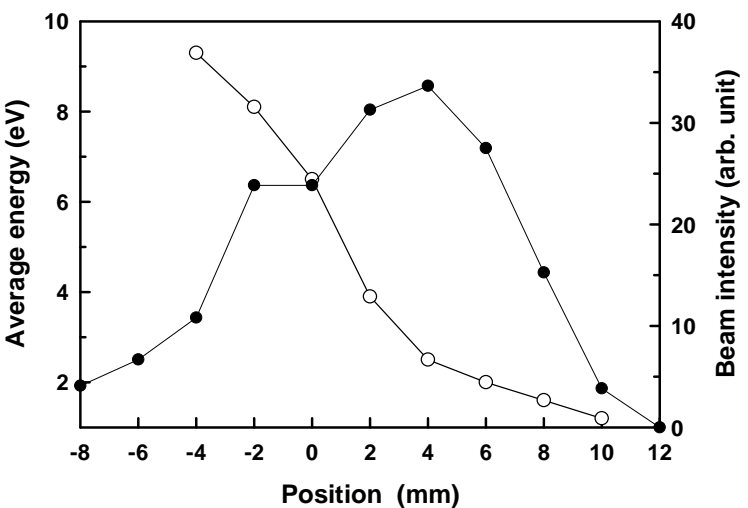

Fig. 3 Average energy and flux distributions over the sample surface. $\bigcirc$ : Average energy and $\bullet$ : Beam intensity

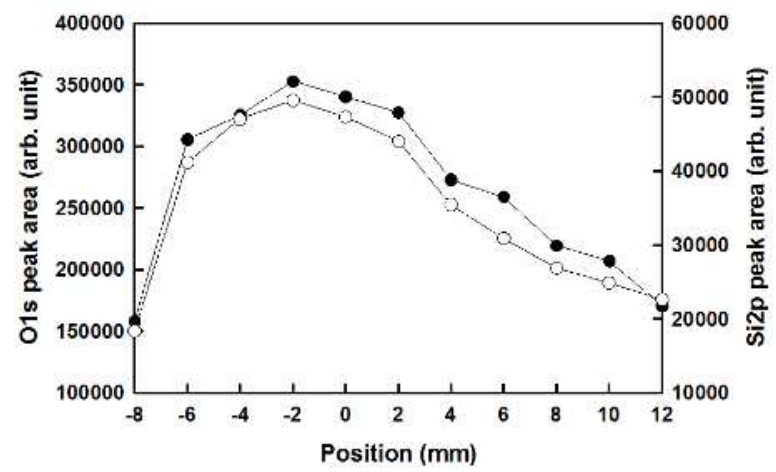

Fig. 4 Comparison of Si2p and O1s SR-PES signal intensities on the sample surface. $\mathrm{O}: \mathrm{Si} 2 \mathrm{p}$ and $\bullet: \mathrm{O} 1 \mathrm{~s}$

result clearly indicated that the accommodation coefficient of O-atom at Si-DLC surface has strong energy dependence. The Si2p signal intensity distribution also shows the similar distribution with O1s which suggests the $\mathrm{O}$-atom is chemically bonded with $\mathrm{Si}$ atoms and remains at DLC surface. This result was supported by the fact that the $\mathrm{C}$ atoms in DLC was removed by forming volatile products such as $\mathrm{CO}$ and $\mathrm{CO}_{2}$ [7].

Figure 5 shows the SR-PES Si2p core level of the Si-doped DLC at different positions normalized by the peak intensity at $100.8 \mathrm{eV}$. The relative intensity of the peak at $103.7 \mathrm{eV}$ depends on the position on the sample surface. The chemical shifts of $\mathrm{Si} 2 \mathrm{p}$ with respect to metallic Si was reported as follows; $\mathrm{Si}_{2} \mathrm{O}(+1.0 \mathrm{eV}), \mathrm{SiC}$ (+1.1 eV), $\mathrm{SiO}(+2.0 \mathrm{eV}), \mathrm{Si}_{2} \mathrm{O}_{3}(+3.0 \mathrm{eV})$ and $\mathrm{SiO}_{2}(+4.0$ eV) [8]. The chemical shift of $2.9 \mathrm{eV}$ shown in Fig. 5 reflects the conversion from $\mathrm{SiC}$ structure to $\mathrm{SiO}_{2}$ 


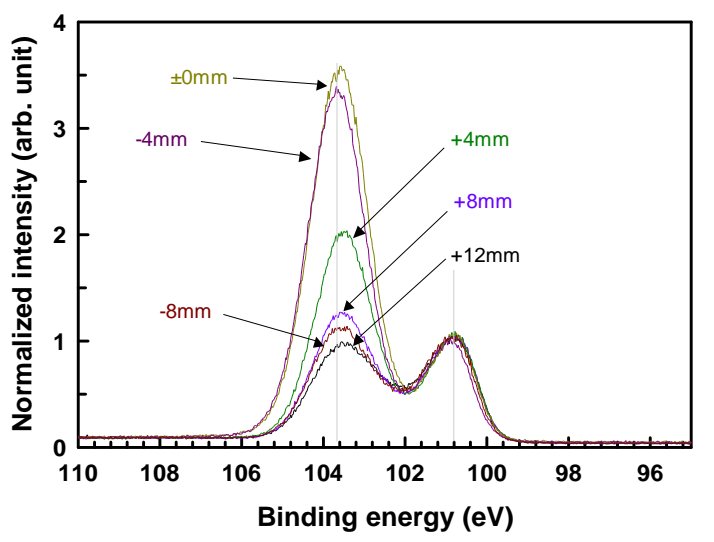

Fig. 5 Change in Si2p SR-PES spectra measured at the sample surfaces from -8 to $+12 \mathrm{~mm}$ positions.

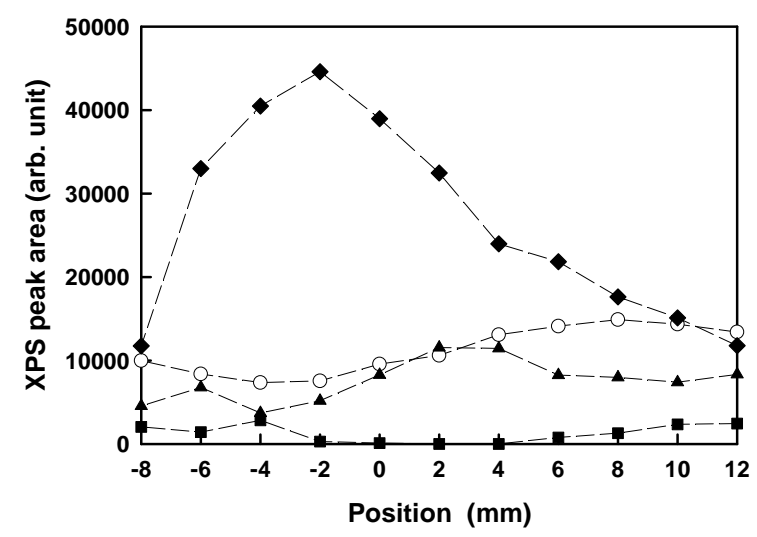

Fig. 6 Peak deconvolution results of Si2p SR-PES spectra over the $\mathrm{O}$-atom beam-exposed sample surface. $\mathrm{O}: \mathrm{SiC}$, $\mathrm{SiO}, \boldsymbol{\Delta}: \quad \mathrm{Si}_{2} \mathrm{O}_{3}, \diamond: \mathrm{SiO}_{2}$.

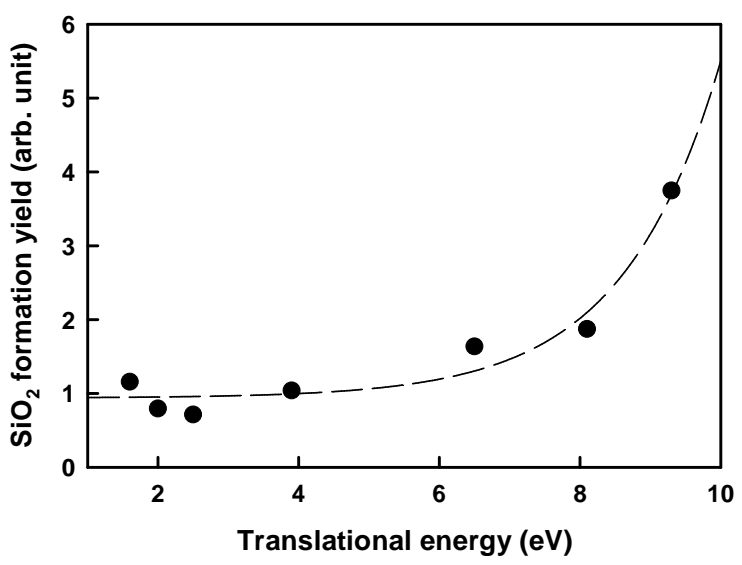

Fig. 7 Translational energy dependence on the reaction yield of O-atom with $\mathrm{Si}$ in DLC. The dashed line is the regression result.

structure by $\mathrm{O}$-atom reaction. This result was supported by the fact that the native oxide of $\mathrm{SiC}$ is $\mathrm{SiO}_{2}$. The
Si2p peaks were deconvoluted by 5 sub-peaks consisting of $\mathrm{SiC}, \mathrm{SiO}_{\mathrm{x}}(\mathrm{x}=0,1,1.5$ and 2$)$. The results of deconvolution are shown in Fig. 6. It was clearly indicated that the $\mathrm{SiO}_{2}$ intensity has a summit at the -2 $\mathrm{mm}$ position which is low-flux but high-energy O-atom-exposed region. In contrast, sub-oxide $\left(\mathrm{Si}_{2} \mathrm{O}_{3}\right)$ is distributed at the $+4 \mathrm{~mm}$ position where low-energy O-atoms reacted. It was also observed that the peak intensity of suboxides decreased with increasing the collision energy of incoming O-atom. The peak intensity of $\mathrm{SiO}_{2}$ is rapidly increased at the position of +4 $\mathrm{mm}$ which corresponds to the translational energy of 2.5 $\mathrm{eV}$ even though the flux decreased (see Fig.3). It was thus concluded that the formation of $\mathrm{SiO}_{2}$ by the $\mathrm{O}$-atom collision is promoted by the translational energy higher than $2.5 \mathrm{eV}$ at room temperature.

The reaction yield of $\mathrm{O}$-atom with $\mathrm{Si}$-atom in DLC was evaluated by the SR-PES $\mathrm{SiO}_{2}$ signal divided by O-atom flux. The results are shown in Fig. 7. It is clearly indicated that the reaction yield of O-atom increased exponentially with increasing translational energy. The reaction yield at $9 \mathrm{eV}$ is 4 times greater than that at $2 \mathrm{eV}$. The Si-atoms in DLC are considered to bond with neighboring $\mathrm{C}$ atoms, which form $\mathrm{Si}-\mathrm{C}$ bonding. Oxidation of $\mathrm{Si}-\mathrm{C}$ bonding has been studied relating to the SiC-based devices. However, a limited number of publications are available for $\mathrm{SiC}$ oxidation by $\mathrm{O} / \mathrm{O}_{2}$ beam. Takahashi et al studied the initial oxidation reaction of $4 \mathrm{H}-\mathrm{SiC}(0001)$ with $0.5 \mathrm{eV} \mathrm{O}$ beam. They reported that the $\mathrm{O}_{2}$ molecule is immediately dissociated at the $\mathrm{SiC}(0001)$ surface and O-atoms are inserted into $\mathrm{Si}-\mathrm{Si}$ backbond to form stable oxide [9]. The translational energy dependence on the $\mathrm{O} / \mathrm{O}_{2}$ reactions with $\mathrm{SiC}$ has not been reported to date. The result of this work suggests that the $\mathrm{SiO}_{2}$ formation on the $\mathrm{Si}$ atoms in DLC is promoted by the O-atom translational energy greater than $5 \mathrm{eV}$ which is the collision environment in LEO. Moreover, $\mathrm{N}_{2}$ molecule in LEO simultaneously collides to DLC at $9 \mathrm{eV}$ because of its larger mass. The simultaneous collision of $5 \mathrm{eV}$ $\mathrm{O}$-atom and $9 \mathrm{eV} \mathrm{N}_{2}$ could accelerate the oxidation of Si-atom at DLC surface if $\mathrm{N}_{2}$ collision provides the energy to overcome the potential barrier of the oxidation reaction (collision-induced oxidation). The presence of this synergistic effect is still to be discovered. 
Journal of Surface Analysis Vol.20, No. 3 (2014) pp. 221-225

K. Yokota et al. A Synchrotron Radiation Photoelectron Spectroscopic Study on the Oxidation of Si in Diamond-like Carbon Film by Hyperthermal O-atom Beam

\section{Conclusions}

The translational energy dependence on the oxidation efficiency of Si atom in DLC was studied with hyperthermal O-atom beams. It was confirmed that the oxidation yield of $\mathrm{O}$-atom with $\mathrm{Si}$ increase exponentially with the collision energy of $\mathrm{O}$-atom in the range of 2 to 9 $\mathrm{eV}$. It was also examined that the amount of suboxides decreased with increasing the collision energy of incoming O-atom above $4 \mathrm{eV}$. It was concluded that formation of $\mathrm{SiO}_{2}$ by the collision of energetic $\mathrm{O}$-atom in LEO is promoted with the orbital velocity of spacecraft at $8 \mathrm{~km} / \mathrm{s}$, which corresponds to the collision energy of 5 $\mathrm{eV}$.

\section{Acknowledgments}

A part of this study was supported by the Grant-in-Aids from the Japan Society for Promotion of Science (\#23360378 and \#25289307).

\section{References}

[1] J. C. Yang, K. K. de Groh, MRS Bulletin 35, 19 (2010).
[2] L. J. Leger, NASA TM-58246 (1982).

[3] V. Srinivasan, B. A. Banks Eds. in Materials degradation in low earth orbit (LEO), TMM, Warrendale, PA (1990).

[4] E. Miyazaki, M. Tagawa, K. Yokota, R. Yokota, Y. Kimoto, J. Ishizawa, Acta Astronaut. 66, 922 (2010).

[5] M. Tagawa, K. Yokota, A. Yoshigoe, Y. Teraoka, T. Shimura, Appl. Phys. Lett. 88133512 (2006).

[6] K. Yokota, S. Yasuda, A. Mizutani, M. Tagawa, Jpn. J. Appl. Phys. 52, 038002 (2013).

[7] M. Tagawa, K. Kishida, K. Yokota, K. Matsumoto, A. Yoshigoe, Y. Teraoka, J. Zhang, T. K. Minton, in Protection of Materials and Structure in a Space Environment, J. Kleiman, M. Tagawa, Y. Kimoto Eds., pp.547, Springer, Heidelberg (2013).

[8] J. F. Moulder, W. F. Stickle, P. E. Sobol, K. D. Bomben, in Handbook of X-ray Photoelectron Spectroscopy, PHI, Eden Prairie, MN (1995).

[9] S. Takahashi, S. Hatta, A. Yoshigoe, Y. Teraoka, T. Aruga, Surf. Sci. 603, 221 (2009).. 